\title{
DESREGULACIÓN FINANCIERA Y CRISIS ECONÓMICA GLOBAL
}

\section{FINANCIAL DEREGULATION AND GLOBAL ECONOMIC CRISIS}

\section{Adrián Alejandro Flores Konja *}

Docente Principal de la Facultad de Ciencias Contables- UNMSM

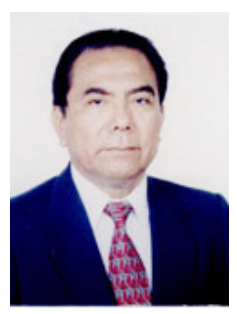

COlaborador : Manuel Alberto Hidalgo Tupia **

Docente Auxiliar de la Facultad de Ciencias Contables - UNMSM

[Recepción: Agosto de 2011/ Conformidad: Octubre de 2011]

\section{RESUMEN}

En este artículo se revisan los cambios en las normas y prácticas en el ámbito financiero a nivel global, y se lo relaciona con la crisis financiera de los Estados Unidos de 2007 y la actual crisis económica europea. Partiremos del análisis del Informe de la Comisión Federal Investigadora de la Crisis Financiera de los Estados Unidos, de Enero de 2011.Trataremos de establecer en forma preliminar la relación entre los cambios en las normas, prácticas y políticas clásicas establecidas desde 1933 en los Estados Unidos - y los acontecimientos posteriores.

En especial, se busca relacionar la política de desregulación financiera impuesta a partir de 1971, con el fin del Acuerdo original de Bretton Wood de 1944, en el orden monetario financiero internacional, en especial, la abrogación de 1999 de la Ley Bancaria -- legislación Glass - Steagall -- que separaba al sector financiero ligado a la producción y distribución física, del sector financiero puramente especulativo, que contemplaba además una garantía y protección a los primeros, y severas regulaciones a los segundos. A partir de entonces, la especulación comenzó a desplazar a las actividades financieras ligadas al sector real, como lo atestigua el crecimiento de los derivados financieros.

$\mathrm{Al}$ presente, la crisis financiera norteamericana se ha hecho europea, (Portugal, Irlanda, Grecia y España), y es en realidad la crisis de un conglomerado bancario internacionalizado, y esta complicando las finanzas estatales con un endeudamiento descomunal para salvar al sistema financiero especulativo, con el rechazo de las poblaciones, la crisis política y el riesgo creciente consiguiente.

Palabras clave: Crisis financiera internacional. Desrregulación. Legislación Glass - Steagall. Comisión Angelides. Acuerdos de Bretton Wood. Grupo Inter Alpha. Crisis fiscal. Endeudamiento público.

\begin{abstract}
This article examines the changes in rules and practices in the financial sector globally, and it is related to the financial crisis of 2007 U.S. and European economic crisis. We leave the analysis of the Report of the Federal Commission of Inquiry into the Financial Crisis of the United States, January 2011.Trataremos to establish a preliminary relationship between changes in rules, practices and policies established classic since 1933 in the United States - and subsequent events. In particular, it seeks to link the financial deregulation policies imposed from 1971, to the original Bretton Woods Agreement of 1944, the international monetary and financial order, especially the repeal of the Banking Act 1999 legislation Glass - Steagall - that separated the financial sector linked to the production and physical distribution, speculative financial sector, which provided also a security and protection to the former and the latter severe regulations. Since then, speculation began to displace the financial activities related to the real sector, as evidenced by the growth of financial derivatives. At present, the U.S. financial crisis has made European (Portugal, Ireland, Greece and Spain) and is actually a conglomerate banking crisis internationalized, and this complicated state finances with a huge debt to save the speculative financial system with the rejection of populations, the political crisis and the consequent increased risk.
\end{abstract}

Keyword: International financial crisis. Deregulation. The Glass - Steagall. Angelides Commission. Bretton Wood. Inter Alpha Group. Fiscal crisis. Public debt.

* Doctor en Ciencias Contables y Empresariales- UNMSM, Contador Público Colegiado Certificado. Actual Decano de la Facultad de Ciencias Contables - UNMSM. E- mail: afloreskonja@yahoo.es

** Magister en Ciencias Económicas - UNMSM, Docente de la Facultad de Ciencias Contables - UNMSM.

E- mail: afloreskonja@yahoo.es 


\section{INTRODUCCION}

Las realidades de la globalización no solo se vivirán en los aspectos positivos, como son el incremento del comercio internacional, las inversiones extranjeras directas y el mayor intercambio migratorio y cultural, también se vive en su contraparte problemática, como se ha visto en el caso de la crisis financiera internacional que, produciéndose en los países centrales, repercute de inmediato en los países periféricos. Como lo dijo un gran político peruano: "Cuando hay resfriado en Nueva York, en el Perú es pulmonía”.

Es por eso que se ha hablado de un "efecto contagio" u "efecto dominó", significando que una crisis financiera en un país dentro de un sistema globalizado repercute de inmediato en los demás países. Para poner un ejemplo, la crisis global de 1998, con la llamada crisis rusa y la quiebra del LTCM, nuestro país llegó a una recesión que se extendió hasta el año 2002.

Es por eso que, enfocamos como un sistema la economía globalizada, en el cual la función principal en el orden monetario corresponde a la Reserva Federal de los Estados Unidos y en el financiero al eje bancario Londres - Nueva York.

\section{LOS MERCADOS FINANCIEROS SEGÚN LA TEORIA ECONÓMICA}

Los mercados financieros son los que ponen en contacto a aquellos agentes económicos deficitarios de fondos, (Oferentes de activos financieros) y los excedentarios de fondos (Demandantes de activos financieros); que al entrar en contacto establecen una operación financiera, en la cual el primero es el prestatario y el segundo el prestamista. Por ejemplo, "Saga Falabella", al planear la ampliación de sus tiendas en el interior del Perú, es demandante de fondos, $y$ al emitir instrumentos de corto plazo, es a la vez oferente de Activos financieros. Del otro lado está el Fondo Mutuo "Interfip", como oferente de fondos, que compra dichos instrumentos, como demandante de los Activos financieros. Ambos coinciden en el mercado financiero. Ver Grafico No 1

Es generalmente reconocido que el deficitario aplicará los fondos así obtenidos para financiar especialmente la producción del bien o servicio, o en su defecto, alguna actividad relacionada, como la logística u alguna otra. Esto se puede producir mediante un sistema de intermediación directa, por ejemplo, a través de la Bolsa de Valores o la emisión de

\begin{tabular}{|c|c|}
\hline \multicolumn{2}{|c|}{$\begin{array}{c}\text { Grafico No } 1 \\
\text { Principales sujetos del mercado financiero }\end{array}$} \\
\hline $\begin{array}{l}\text { Demandante de crédito o } \\
\text { fondos } \\
= \\
\text { Oferente de Activos } \\
\text { financieros }\end{array}$ & $\begin{array}{l}\text { Oferente de crédito o } \\
\text { fondos } \\
=\end{array}$ \\
\hline \multicolumn{2}{|c|}{$\begin{array}{l}\text {-Por ejemplo, Saga Falabella es demandante de fondos y a la vez } \\
\text { oferente de Activos financieros (Instrumentos de corto plazo). Por } \\
\text { ejemplo, del otro lado está un Fondo Mutuo - como Oferente de } \\
\text { fondos - a la vez demandante los Activos financieros, como los } \\
\text { que emite Saga. }\end{array}$} \\
\hline \multicolumn{2}{|c|}{-Ambos se encuentran en el Mercado financiero. } \\
\hline
\end{tabular}

148/ QUIPURAMAYoc | Vol. 19(36) 2011 
bonos, o mediante un sistema de intermediación indirecta, como puede ser con la intervención de la entidad bancaria, que también puede intervenir sin tanto protagonismo en el primer tipo. Ver Grafico No 2

Los mercados financieros también se pueden diferenciar por el plazo de las operaciones que ofertan, siendo el mercado monetario el de corto plazo y el mercado de capitales de plazo mayor. Ver Gráfico № 3
Como se notará, la innovación es el Mercado de Futuros y derivados, creado originalmente para dotar de cobertura a los agentes económicos sujetos a los riesgos de mercado de diferente tipo, sin embargo, su desarrollo ha salido totalmente de este objetivo, siendo usado mayormente para la especulación.
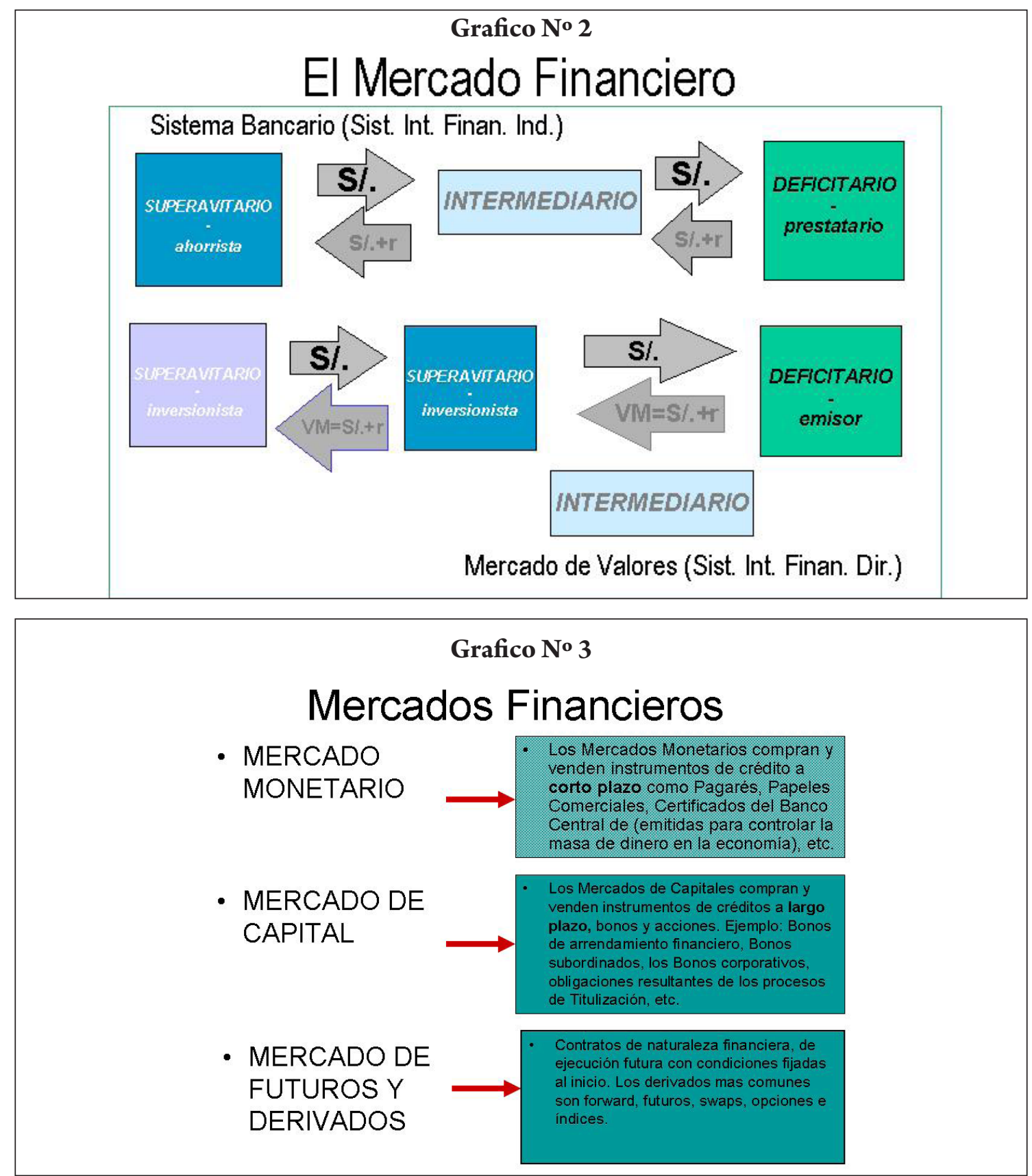


\section{EL PROCESO DE LOS DERIVADOS.}

Los derivados son activos financieros "derivados" - de allí su nombre - de otros activos que si franquean derechos reales sobre activos físicos y ganancias de empresas reales. Existen muchos tipos de derivados: sobre acciones, sobre tipo de cambio, sobre tenencia de moneda extranjera, sobre commodities, etc. En líneas generales, son contratos de compra u opción de compra a determinada fecha en el futuro (por eso se les suele llamar según la modalidad opciones, futuros, forwards, etc.) en el cual se pacta con anticipación el precio de la transacción. Así, llegada la fecha, de acuerdo al precio real de mercado de dicha fecha, habrá un "ganador" y un "perdedor", respecto al precio pactado. Los activos motivo del contrato pueden o no transferirse (por eso se llama opciones), pero la diferencia (ganancia para uno y pérdida para otro) si debe liquidarse en la fecha. Quienes escriben han tratado de hallar una diferencia de fondo entre estos contratos y una apuesta, y no la hemos hallado.

Estas apuestas pueden girar sobre: tipo de cambio, tasas de interés, índices bursátiles, precios de las commodities, etc. En términos formales los derivados generan una "cobertura" o protección contra riesgos de mercado; por ejemplo, una empresa endeudada en dólares y con ingresos en moneda nacional, que puede sufrir perjuicios de una devaluación de su moneda local, puede firmar un contrato forward, lo mismo si depende del abasto de un commoditie, o caso contrario produce y vende un commoditie en mercados muy fluctuantes.

Pero el motivo "cobertura" es cada vez menos frecuente, pues la gran mayoría de los contratantes no son empresas productivas, sino agentes financieros, cuyo motivo básico es la "especulación"; siendo suficientemente grandes o actuando en colusión pueden influir sobre los precios, en mercados oligopolizados.

Es decir, la "especulación" normal - esperar por ejemplo que el precio de las acciones que se posee suba - se ha visto multiplicada por el uso de estos contratos, llevando a los mercados financieros a convertirse, en palabras del premio Nobel de Economía francés Maurice Allais, en un "casino mondiale".

\section{LA DESRREGULACION DE LOS MERCADOS FINANCIEROS Y EL GIRO HACIA LA ESPECULACION, SEGÚN EL INFORME ANGELIDES}

Así pues, estas características originales se han transformado en el transcurrir de los años, presentándose estas dos tendencias interrelacionadas: la desrregulación, partiendo de los Estados Unidos, y el giro hacia la especulación del sistema financiero metropolitano. A consecuencias de la gran crisis de 2008, el Congreso de los Estados Unidos formó en mayo de 2009 una Comisión Federal Investigadora de la Crisis Financiera de los Estados Unidos, convocada "para examinar la crisis economica y financiera que ha afectado a nuestro pais, explicar sus causas al pueblo norteamericano". La Comision fue presidida por el congresista Phil Angelides, y luego de 18 meses, en Enero de 2011, presentó su Informe Final (Ver Figura $\mathrm{N}^{\circ} 1$ ). El Informe Angelides describe el proceso mencionado, identificando claramente las dos tendencias ya citadas, señalándolas como la causa de la crisis financiera.

En el trascurso de esa investigación fueron citados a declarar más de 700 testigos, entre ellos no solo de los gigantes financieros en crisis, sino también los líderes de la Reserva Federal, el Departamento del Tesoro y la Comisión Bancaria y de Valores, entre otras 
agencias regulatorias federales en cuyos ámbitos de responsabilidad descansaba la supervisión directa de las actividades financieras que inexplicablemente nunca detectaron o sospecharon que fueran irregulares.

En mas de 500 páginas de análisis pormenorizado, la Comisión señala respecto a las consecuencias de la crisis:

"Al cierre de este Reporte más de 26 millones de estadounidenses se encuentran sin trabajo, cerca de 4 millones de familias han perdido sus casas en remates y otras cuatro y medio millones han escapado al remate de sus casas pero se encuentran atrasados en el pago de sus hipotecas. Cerca de 11 millones de millones de dólares en capital inmobiliario se han esfumado... El impacto de esta crisis lo resentirá una generación, sin tener a la vista un camino fácil para recuperar el vigor económico."

La Comisión concluye, respecto a las circunstancias y causas de la crisis que:

* “... esta crisis podía haberse evitado, es resultado de la acción humana y de omisiones...."

* “...amplios fallos en la regulación y supervisión financiera fueron comprobadamente devastadores para la estabilidad de los mercados financieros de la nación”.
* “... dramáticos fallos de gobierno corporativo y gestión de riesgos en muchas importantes instituciones financieras del sistema fueron la causa clave de esta crisis".

* “ ... la combinación de préstamos excesivos, inversiones riesgosas y falta de transparencia, pusieron al sistema financiero en curso de colisión"

* “... el gobierno no estaba preparado para esa crisis, y su respuesta inconsistente agravó la incertidumbre y el pánico en los mercados financieros".

* “... hubo una quiebra sistémica en la contabilidad y la ética”

* “... el colapso de los estándares de préstamos hipotecarios y la securitización hipotecaria en trámite levantaron y expandieron la flama del contagio y la crisis"

* "... los derivados over the counter contribuyeron significativamente a la crisis."

* "... las fallas de las agencias clasificadoras de crédito fueron engranajes esenciales en las ruedas de la destrucción financiera."

El Informe advierte que mientras no se corrijan las fallas y omisiones del marco regulatorio del sistema financiero ese tipo de crisis puede repetirse con consecuencias catastróficas de mayor profundidad y costo que las que hemos visto hasta ahora.

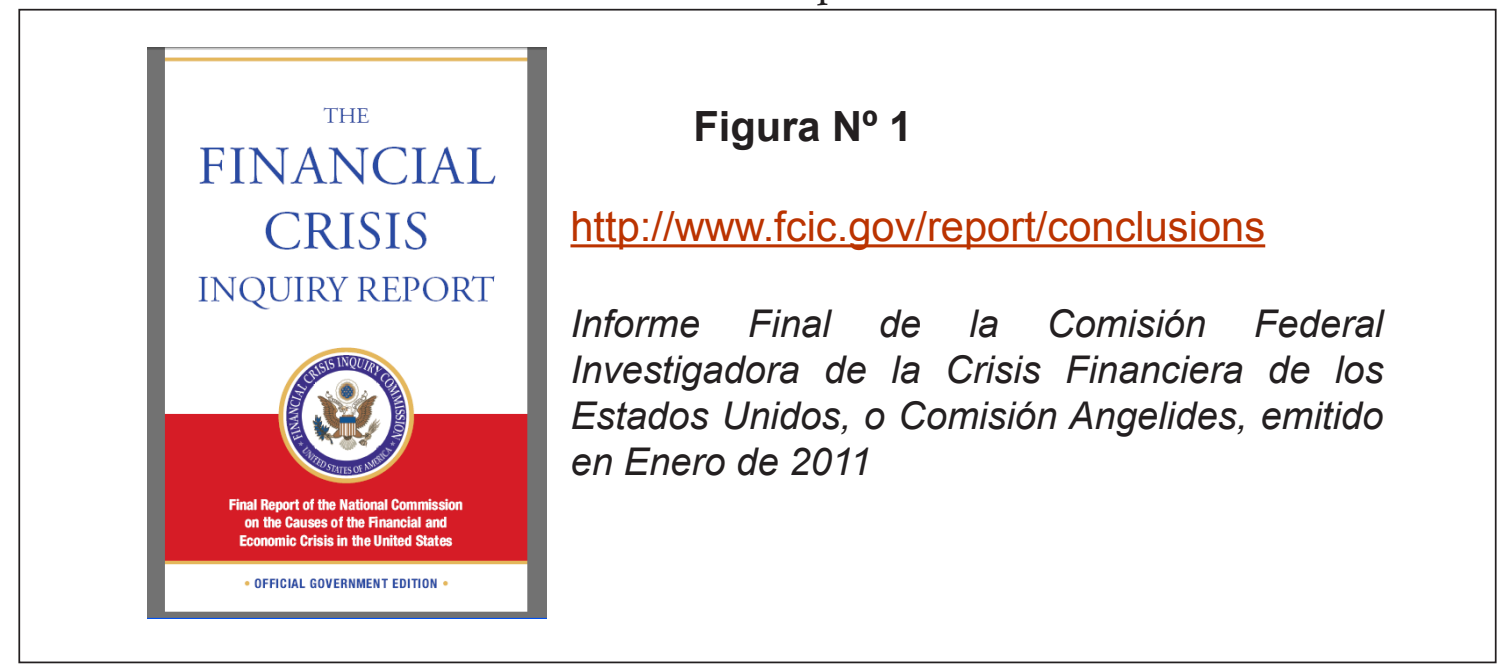


Poniendo en contexto del Informe, debemos remontarnos a 1933, cuando el entonces presidente de los Estados Unidos F.D. Roosevelt establece una serie de medidas para superar la gran crisis de 1929; entre ellas, las contenidas en la Ley Glass - Steagall, Ley Bancaria, que constituyó una separación entre la banca de depósitos - más ligada a la industria y el comercio - y la banca de inversiones, mucho más especulativa, permitiendo el rescate de la primera castigando a su vez la deuda especulativa de la segunda: A esto se unió la inyección de crédito público pero para la reconstrucción de la economía física norteamericana, "rescatando" a la empresa productiva y, de pasada, a los bancos comerciales acreedores. Es decir, todo lo opuesto a la "austeridad." que los organismos internacionales pregonan en la actualidad. Se suspendieron los embargos por leyes expresos, generando plazos de gracia convenientes., a la vez que se forzó a los bancos a castigar los activos especulativos depreciados por el mercado.

La Ley Glass Steagall establece un sistema completo de regulación bancaria, además, la Ley Anti monopolios (Ley Sherman) limitaba la conformación de holdings bancarios. Se limitaba también la presencia de los banqueros en los consejos de administración de las empresas industriales y comerciales.

Pero esto sería incompleto sin la serie de proyectos de infraestructura, como la $\mathrm{Au}$ toridad del Valle del Teenessee, las represas Hoover y Grand Coulee, que fueron ejemplo de proyectos desarrollistas en el mundo entero. Al cabo de algunos años, la economía norteamericano había sido rescatada, y el sistema bancario con ella.

\section{DESENLACE DE LAS TENDENCIAS ECONOMICAS GLOBALES: LA CRISIS ACTUAL DEL SISTEMA DEL DÓLAR}

La desregulación financiera fue impuesta progresivamente a partir de 1971, con el fin del Acuerdo original de Bretton Wood de 1944, en el orden monetario financiero internacional, pero en especial, con la abrogación de la legislación Glass - Steagall en 1999, luego de una campaña de varios lustros, afirmando que esta Ley limitaba el crecimiento y expansión internacional de la banca norteamericana, y su incursión en los derivados financieros. De hecho, la especulación y su nueva ronda con los derivados estuvieron centradas en la City de Londres, y sus sedes europeas, los bancos norteamericanos participaban de la mano de la City con sus sucursales fuera de los EE.UU.; limitados de hacerlo internamente por la Ley Glass - Steagall hasta 1999. Las quiebras de Enron y LTCM fueron la ocasión para que la Reserva Federal "abriese el caño" de la emisión primaria a los bancos adictos a los derivados, que a partir de entonces sobreabundaron en sus operaciones con derivados.

Como hecho significativo, la primera consecuencia del la abrogación de la Ley Glass Steagall fue la consumación legal de la fusión del Citigroup y el Traveller. A partir de entonces, las actividades financieras puramente especulativas, es decir, no ligadas en forma directa a la producción y al intercambio, comenzaron a desplazar a las ligadas al sector real, como lo atestigua el crecimiento de los derivados financieros. Por ejemplo, en la actualidad menos del $2 \%$ de las transacciones financieras internacionales tienen alguna relación con el comercio de bienes tangibles. Ver Gráfico No 4 
TRANSACCIONES DE DIVISAS COMPOSICIÓN PORCENTUAL DE LOS MONTOS EN US\$
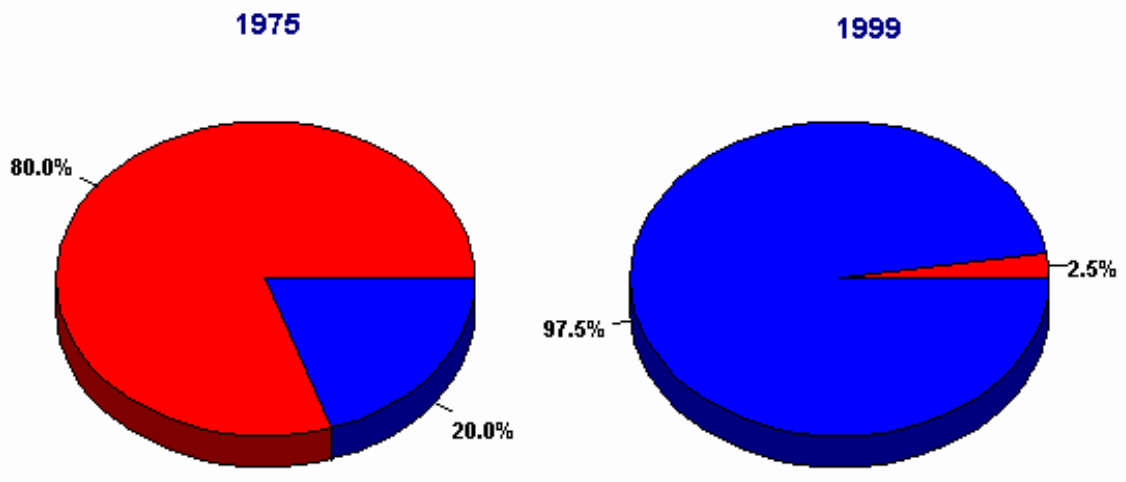

\section{ఐREALES ĐESPECULATIVAS}

Fuente: Bernard Lietaer, UC at Berkeley, USA

EL Informe Angelides da algunos detalles de este proceso, como lo atestiguan las citas que alcanzamos:

"La crisis alcanzó proporciones sísmicas en septiembre de 2008 con la caída del Lehman Brothers y el inminente colapso del gigante asegurador American International Group AIG. El pánico creció por una falta de transparencia de las hojas de balance de las mayores instituciones financieras."

"Mas de 30 años de desregulación y de confianza en la autorregulación por las instituciones financieras, promovidas por el ex presidente de la Reserva Federal Alan Greenspan y otros, apoyados por administradores gubernamentales y congresistas, $y$ activadas activamente por la poderosa industria financiera en toda ocasión, desnudaron de las salvaguardias claves que pudieron impedir la catástrofe. "

"Y el apalancamiento fue usualmente escondido en posiciones de derivados, en entidades fuera de balance y a través del ma- quillaje de los reportes financieros disponibles para el público inversionista."

"La aprobación de la legislación en el 2000 para desterrar la regulación de los gobiernos federal y estatal de los derivados over the counter (OTC) fue el punto de inflexión clave en la marcha sobre la crisis financiera."

"Desde firmas financieras hasta corporaciones, agricultores e inversionistas, los derivados fueron usados para cubrirse o especular sobre los cambios de precios, tasas, o índices, o también en eventos tales como un potencial incumplimientos de deudas. Así, sin ninguna supervisión, derivados OTC crecieron fuera de control y fuera de la vista, creciendo hasta 673 millones de millones en montos nocionales. Este reporte explica el apalancamiento incontrolable. Las faltas de transparencia y de requerimiento de capital y colaterales; Especulación; Interconexión entre firmas; y concentración de riego en el mercado" 
Como el Informe Angelides documenta, esta situación fue la que llevó a la crisis en los Estados Unidos, en los sucesivos incidentes, como la Crisis de 1987, y la crisis de la quiebra del LTCM, de 1998, y las reverberaciones en el exterior, sucesivas, como la crisis Rusa, la Crisis Mexicana, etc. hasta reventar en una crisis en la propia metrópoli (Ver Cuadro No 5 ).

\section{Cuadro $\mathrm{N}^{\circ} 5$}

\section{Cronología de las crisis financieras de mayor impacto sistémico}

\begin{tabular}{|ll|}
\hline 1987 & Octubre Crisis bursátil de los EEUU. Lunes negro. \\
\hline 1994 & Crisis bancaria mexicana \\
\hline 1997 & Julio. Crisis asiática \\
\hline 1998 & Crisis de la deuda Rusa y desplome de la Bolsa de Nueva York (Septiembre) \\
\hline $\mathbf{1 9 9 8}$ & Quiebra del Long Term Capital Management \\
\hline $\mathbf{2 0 0 1}$ & Crisis argentina \\
\hline $\mathbf{2 0 0 1}$ & Diciembre. Quiebra fraudulenta del gigante energético norteamericano Enron \\
\hline $\mathbf{2 0 0 7}$ & Quiebra del mercado hipotecario de los EEUU \\
\hline $\mathbf{2 0 0 8}$ & $\begin{array}{l}\text { Quiebra de Lehmman Brothers e inestabilidad de la banca de inversión de los } \\
\text { EEUU. Rescate fiscal a los bancos de inversión. }\end{array}$ \\
\hline 2011 & Crisis económica y política europea por el fracaso de los rescates bancarios. \\
\hline
\end{tabular}

Como lo dijo en 1998 en su momento el presidente de la Reserva Federal, Alan Greenspan, desde la quiebra del LTCM, se iba a evitar la quiebra del sistema con un "muro de dinero", es decir, con emisión monetaria al extremo para sostener los rescates.

Pero peor fue el "remedio" de los rescates que la enfermedad de las quiebras bancarias. Al presente, la crisis financiera europea en curso, centrada aparentemente en Portugal, Irlanda, Grecia y España, es la crisis de un sistema bancario internacionalizado, y esta complicando las finanzas estatales con un endeudamiento descomunal para salvar al sistema financiero especulativo, con el rechazo de las poblaciones y la crisis política consiguiente.
Es decir, los rescates multimillonarios, no solo en Estados Unidos, sino también en Europa, además de complicar la ya deteriorada situación fiscal de los Estados Unidos en especial y Europa en el mismo sentido, están yendo a convertirse en una crisis política y monetaria.

El Informe plantea la pregunta fundamental: “¿Cómo fue posible que en el 2008 los Estados Unidos se viera forzado a escoger entre dos alternativas, -igualmente dolorosas-, entre el riesgo de un colapso total de la economía y del sistema financiero, o inyectar millones de millones de dólares al sistema financiero y a un grupo de compañías, mientras millones de estadounidenses perdían sus trabajos, sus ahorros y sus casas?" 
La ultima trinchera es el poder de manejar la Reserva Federal como prestamista de ultimo recurso, pero este poder reside en la capacidad y deseo de países como China y Japón de seguir honrando el papel actual de Estados Unidos como el centro monetario, adquiriendo masivamente activos denominados en dólares para sus reservas.

Cuando la realidad o la voluntad pongan un freno a esta política; el sistema del dólar se caería como un castillo de naipes.

Entretanto, el efecto inflacionario de la política de la Reserva Federal del "muro de dinero" esta desbordando el campo financiero para atacar los mercados reales de alimentos, y combustibles, entre otros.

\section{CONCLUSIONES Y RECOMENDACIONES}

Es necesario que las Facultades de Contabilidad de nuestra región asuman la tarea de analizar las características, causas y consecuencias para nuestros países de la actual crisis financiera internacional, recurriendo a un enfoque crítico y socialmente responsable.

Es necesario que las Facultades de Contabilidad de la región participen activamente en difundir las características e implicancias de la crisis financiera internacional y sus efectos en nuestros países, con miras a la formulación de políticas de solución.

Es necesario que las Facultades de Contabilidad de nuestra región asuman la tarea de analizar los aspectos profesionales y éticos de la actual crisis financiera internacional,
Es necesario que los profesionales contables y de las ciencias empresariales en general analicen los detalles contenido en el Informe Angelides, por su importancia científica y deontológica.

\section{REFERENCIAS BIBLIOGRÁFICAS}

1. LARRAÍN B, Felipe \& SACHS, Jeffrey D. Macroeconomía en la economía global. Segunda Edición. Editorial Prentice Hall y Pearson Educación S.A., Buenos Aires - Argentina, 2002.

2. Villacorta Cavero, Armando. Productos y Servicios Financieros. Operaciones bancarias.

3. Sitio electronico de la Comisión Federal Investigadora de la Crisis Financiera de los Estados Unidos. http: / / www.fcic. gov/report/conclusions

4. "The Financial Crisis Inquiry Report", Editorial Public Affairs, Perseus Books Group, enero de 2011. Informe Final de la Comisión Federal Investigadora de la Crisis Financiera de los Estados Unidos, o Comisión Angelides, emitido en Enero de 2011

5. Ley Galls Steagall. En: Wikipedia.

6. http://es.wikipedia.org/wiki/Ley_ Glass-Steagall. Consultado el 18/03 $/ 2011$.

7. Hernández Haddad, Humberto ${ }^{*} \mathrm{El}$ Reporte Angelides 1 marzo 2011 http:// macroeconomia.com.mx/2011/03/ el-reporte-angelides/ Consultado el $15 / 03 / 2011$ 